\title{
MOLECULAR SIGNATURE OF nIDNA-ITS MARKER IN ISODON RUGOSUS (LAMIACEAE)
}

\author{
Devendra Kumar Srivastava ${ }^{1 *}$, Pekhna Bansal ${ }^{1}$, Pradeep Kumar Singh ${ }^{1}$, Manjit Inder Singh \\ SAGGOO $^{2}$
}

${ }^{1}$ Eternal University, Department of Botany, Baru-Sahib, 173101 Himachal Pradesh, India

${ }^{2}$ Punjabi University Patiala, Department of Botany, 147002 Punjab, India

*Corresponding author. E-mail: devsrivastv@rediffmail.com, devsrivastv@gmail.com

\begin{abstract}
Srivastava D.K., Bansal P., Singh P.K., Saggoo M.I.S., 2021: Molecular signature of $n r D N A-I T S$ marker in Isodon rugosus (Lamiaceae). - Botanica, 27(1): 53-61.

Molecular features of $n r D N A-I T S$ sequences in medicinal plant Isodon rugosus were assessed with universal ITS-1 and ITS-2 primers. ITS sequences of 46 Isodon species were defined for their respective ITS-1, 5.8S and ITS-2 regions through in-silico datamining and analysis. Only in 32 species, sequence of all the three defined regions was complete, while in the rest of 14 species was partial. Isodon rugosus revealed five genic regions, namely, $18 S$ rRNA gene (partial sequence, > 1....38), ITS-1 (complete sequence, > 39....236), 5.8S rRNA gene (complete sequence, $>237 \ldots . .400)$, ITS-2 (complete sequence, $>401 \ldots . .607$ ) and large subunit 26S rRNA gene (partial sequence, $>608 \ldots . .672$ ). The sequence regions were recorded with variable sites (VS), singleton (ST) and species-specific parsimonious-informative (PI) sites. The information was also collected in a phylogenetic tree obtained using the maximum likelihood (ML) method.
\end{abstract}

Keywords: Isodon rugosus, Lamiaceae, $n r D N A-I T S$ marker.

\section{INTRODUCTION}

The genus name Isodon (Schrad. ex Benth.) Spach comes from the Greek word 'isos' means equal and 'odous' (or odontos) means tooth (BENTHAM, 1832; SpACH, 1840). It consists of more than 150 species (LiU et al., 2017), distributed chiefly in tropical to temperate East Asia and tropical Africa (MABBERley, 2008; Chen et al., 2017). In India, the genus is represented with nearly 16 species (including one variety), mainly from the Himalayas, Western Ghats, and Nilgiri regions (Sharma, 2018). Isodon rugosus (Wall. ex Benth.) Codd. is an essential indigenous member of Indian Lamiaceae. Species is usually considered quite variable floristically due to obscure molecular identity, taxonomic relationship at inter and intracontinental level, and thus, studied under different names: Isodon plectranthoides, Lumnitzera densiflora, Ocimum densiflorum, Plectranthus rugosus and Rabdosia rugose.
DNA based markers such as $n r D N A-I T S, r b c L$, matK, etc. are reliable, reproducible and unaffected by the physical form and environment. They provide a convenient tool for identifying medicinal plants, crude drugs and even commercial products (SRIVASTAVA \& SAGgoo, 2014; Zhou et al., 2014). Among the different DNA markers, $n r D N A-I T S$ has been supported as a remarkable marker where plastid markers fail to discriminate the plants at the species level (Hollingsworth, 2011). Therefore, nrDNA-ITS was chosen for study in the plant Isodon rugosus. This research is also in addition to our previous work in the plant I. rugosus, where the molecular autograph of plastid marker/gene (matK) was studied (SRIVASTAVA et al., 2020). The present study was undertaken to provide alternative DNA marker information in the taxon I. rugosus that could be employed along with other marker/s for its molecular identification and barcoding. 


\section{MATERIALS AND METHODS}

Plant leaf material was used from the same specimen of I. rugosus (IRBSA-01), which has been studied previously by SRIVASTAVA et al. (2020) in the field population of Baru-Sahib valley of Himachal Pradesh (India). Except for primer pairs $18 S$ (forward) ITSU1: 5'-GGAAGKARAAGTCGTAACAAGG-3' and $26 S$ (reverse) ITS-U4: 5'-RGTTTCTTTTCCTCCGCTTA-3' (CHENG et al., 2015), genomic DNA isolation (CHASE \& Hills, 1991), software applications DNAMAN version 7.0 (Lynnon Biosoft Corporation, USA), MEGA version 7.0 (KumAR et al., 2016) and ITS sequence analysis methodology were similar to that followed in previous of mat $k$ sequence study (SRIVAstava et al., 2020). Phylogeny with maximum parsimony (MP) criterion was avoided due to inconsistency with low scores. Sequence boundaries of different regions (i.e. ITS-1, 5.8S and ITS-2) were determined by comparing the aligned sequences with the sequences of other related species of Isodon registered in NCBI GenBank (Table 1). The complete sequence of the ITS region was deposited at NCBI GenBank (accession No. MH931390).

\section{RESULTS AND DISCUSSION}

The sequence amplified by the primers $18 S$ (forward) ITS-U1 and $26 S$ (reverse) ITS-U4 showed the overall length of amplified $n r D N A$-ITS regions with 569 nucleotides (excluding partial sequences of $18 S$ and $26 S$ rRNA gene). The ITS sequences of 46 different Isodon spp. from the BLASTn result were retrieved from the NCBI GenBank (Table 1) and analysed with a sequence of I. rugosus (MH931390).

In-silico datamining showed only 32 Isodon species with complete $n r D N A-I T S$ sequences, out of which nine species (accessions No. KF032259.1,

Table 1. Isodon species from the BLASTn result and their respective GenBank accession numbers

\begin{tabular}{|c|c|c|c|c|c|}
\hline No. & Species (abbreviations) & Gene Bank No. & No. & Species (abbreviations) & Gene Bank No. \\
\hline 1. & Isodon rugosus $(\mathrm{I}-1)^{*}$ & MH931390 & 25. & I. loxothyrsus (I-25) & FJ593381.1 \\
\hline 2. & I. adenanthus (I-2) & FJ593355.1 & 26. & I. lungshengensis (I-26) & FJ593382.1 \\
\hline 3. & I. adenolomus (I-3) & FJ593356.1 & 27. & I. megathyrsus (I-27)\# & MH117581.1 \\
\hline 4. & I. amethystoides (I-4)\# & KF032259.1 & 28. & I. melissoides (I-28) & FJ593385.1 \\
\hline 5. & I. anisochilus (I-5) & KF855420.1 & 29. & I. nervosus (I-29)\# & KF032257.1 \\
\hline 6. & I. brachythyrsus (I-6) & KF855427.1 & 30. & I. oresbius (I-30) & KF855434.1 \\
\hline 7. & I. bulleyanus (I-7) & FJ593358.1 & 31. & I. parvifolius (I-31) & KF855425.1 \\
\hline 8. & I. coetsa $(\mathrm{I}-8) \#$ & KM877351.1 & 32. & I. pharicus (I-32) & KF855430.1 \\
\hline 9. & I. dawoensis (I-9) & KF855429.1 & 33. & I. phyllostachys (I-33) & FJ593388.1 \\
\hline 10. & I. effuses (I-10) & KF855444.1 & 34. & I. pleiophyllus (I-34) & FJ593389.1 \\
\hline 11. & I. enanderianus (I-11)\# & JQ389514.1 & 35. & I. rosthornii (I-35) & FJ593390.1 \\
\hline 12. & I. eriocalyx $(\mathrm{I}-12)$ & FJ593364.1 & 36. & I. rubescens $(\mathrm{I}-36) \#$ & KF032249.1 \\
\hline 13. & I. flabelliformis (I-13) & FJ593365.1 & 37. & I. rugosiformis (I-37) & FJ593391.1 \\
\hline 14. & I. flavidus (I-14) & FJ593366.1 & 38. & I. scoparius (I-38) & FJ593392.1 \\
\hline 15. & I. flexicaulis (I-15) & FJ593367.1 & 39. & I. sculponeatus (I-39) & FJ593393.1 \\
\hline 16. & I. forrestii $(\mathrm{I}-16)$ & FJ593368.1 & 40. & I. serra $(\mathrm{I}-40) \#$ & KF032262.1 \\
\hline 17. & I. gesneroides (I-17) & FJ593369.1 & 41. & I. setschwanensis (I-41) & KF855412.1 \\
\hline 18. & I. glutinosus (I-18) & FJ593370.1 & 42. & I. shikokianus (I-42) & KF855449.1 \\
\hline 19. & $\begin{array}{l}\text { I. grandifolius var. } \\
\text { atuntzeensis (I-19) }\end{array}$ & FJ593371.1 & 43. & I. shikokianus var. occidentalis (I-43) & KF855450.1 \\
\hline 20. & I. inflexus (I-20)\# & KF032266.1 & 44. & I. taliensis(I-44) & FJ593394.1 \\
\hline 21. & I. irroratus (I-21) & KF855439.1 & 45. & I. wikstroemioides (I-45) & FJ593396.1 \\
\hline 22. & I. japonicas $(\mathrm{I}-22) \#$ & KF032254.1 & 46. & I. xerophilus (I-46) & FJ593397.1 \\
\hline 23. & I. leucophyllus (I-23) & KF855421.1 & 47. & $\begin{array}{l}\text { Rabdosia setschwanen-sis var. yungshengensis } \\
(\mathrm{I}-47)^{* *}\end{array}$ & KF855436.1 \\
\hline 24. & I. longitubus (I-24) & KF855445.1 & 48. & $\begin{array}{l}\text { Mentha suaveolens }{ }^{* * *} \\
\text { M. canadensis }\end{array}$ & $\begin{array}{l}\text { AF369161; } \\
\text { KC473228 }\end{array}$ \\
\hline
\end{tabular}

*Query taxon, **Rabdosia setschwanensis var. yungshengensis - Syn. - Isodon setschwanensis, ***out group species, \# sequences in nine taxa already defined for their respective regions in NCBI databases. 
KM877351.1, JQ389514.1, KF032266.1, KF032254.1, MH117581.1, KF032257.1, KF032249.1 and KF032262.1) were observed with defined regions (e.g. ITS-1, 5.8S, ITS-2), which were originally provided in the NCBI database (Table 1). Remaining all the species that were not defined by their respective regions hitherto is now defined and presented in Table 2. The information regarding accession numbers, length, sequence span and GC content [calculated as $\mathrm{G}+\mathrm{C} \%=\{(\mathrm{G}+\mathrm{C}) /(\mathrm{A}+\mathrm{T}+\mathrm{G}$ $+\mathrm{C})\} \times 100]$ of the different ITS regions (i.e. ITS-1, $5.8 S$ and ITS-2) of various Isodon species are provided in Table 2.

Amplified sequence in $n r D N A-I T S$ sequence in I. rugosus was reported with five regions: $18 \mathrm{~s}$ ribosomal RNA gene (partial sequence, $>1 \ldots . .38$ ), ITS-1 (complete sequence, $>39 \ldots . .236$ ), 5.8S rRNA gene (complete sequence, $>237 \ldots . .400$ ), ITS-2 (complete sequence, $>401 \ldots . .607)$ and large sub unit (26S $r R N A$ gene partial sequence, $>608 \ldots . .672$ ).

\section{ITS-1 region}

The sequence of ITS-1 in I. rugosus was observed with a length of $198 \mathrm{nt}$ bases. Excluding the sites with missing (or ambiguous) data and gaps produced significant alignment (SA) in the ITS-1 region at 198 nt sites $(>39 \ldots . .236)$. From the aligned positions of the ITS-1 region of 47 taxa, in I. rugosus, 49 sites were variable (VS), 30 sites were singleton (ST) and 19 sites of which were observed as parsimonious-informative (PI). Distribution of informative sites with their respective nucleotide bases (e.g. A, T, G or C) was observed to be positioned at 60 (C), 67 (A), 77 (C), 82 (T), 84 (C), 94 (A), 100 (C), 106 (C), 114 (G), $116(\mathrm{G}), 147$ (A), $166(\mathrm{~A}), 167$ (T), 180 (C), 183 (C), $205(\mathrm{G}), 213$ (C), 221 (G) and 229 (C) (Table 3). The GC content in the ITS-1 region of I. rugosus was $66.16 \%$. The spanning length of the ITS- 1 region varied from $194 \mathrm{nt}$ to $198 \mathrm{nt}$, with a more frequent (in 44 species) occurrence of $196 \mathrm{nt}$ (Table 2). Length variation was observed in three taxa, namely, I. rugosus (> 39....236; $198 \mathrm{nt})$; I. brachythyrsus $(>01 \ldots . .194$; $194 \mathrm{nt})$ and $I$. coesta $(>33 \ldots . .227 ; 195 \mathrm{nt})$.

\section{$5.8 S$ region}

The spanning length of the $5.8 S$ region of $n r D N A$ in I. rugosus was $164 \mathrm{nt}$ bases $(>237 \ldots . .400)$. In $I$. rugosus, out of 164 aligned positions, four sites were variable, three sites were singleton, and one (1) site was parsimonious informative. The parsimonious informative site in the aligned sequences of the $5.8 \mathrm{~S}$ region was observed (Table 4) at position 237 (A). The GC content $(\mathrm{G}+\mathrm{C} \%)$ in the region was $54.88 \%$. In 45 species, the length of the $5.8 \mathrm{~S}$ region was observed with a similar value of $164 \mathrm{nt}$ bases, while GC content was identical in 40 species (Table 2). One species, i.e. I. phyllostachys (I-33; > 197.....359), was reported with single nucleotide deletion, making its spanning length of $163 \mathrm{nt}$ bases (Table 2). The GC content differed in six species, namely, I. coesta (I-08, G + C\%: 55.49), I. inflexus (I-20, $\mathrm{G}+\mathrm{C} \%$ : 54.27), I. lungshengensis (I-26, GC\%: 55.49), I. parvifolius (I-31, G + C\%: 54.27), I. phyllostachys (I-33, G + C\%: 55.21) and I. shikokianus var. occidentalis (I-43, G + C\%: 55.49).

\section{ITS-2 region}

Alignment in the ITS-2 region of I. rugosus was significant at $207 \mathrm{nt}$ positions $(>401 \ldots . .607)$. There were $38 \mathrm{nt}$ sites, which were variable, 26 sites were singleton, and 12 sites were parsimonious informative. Parsimonious informative sites (Table 4) were observed at positions 418 (A), 419 (C), $424(\mathrm{G}), 425$ (C), 429 (C), 433 (G), 450 (T), 502 (C), 551 (C), 558 (T), 565(T) and $568(\mathrm{C})$. The GC richness was reported as $68.12 \%(\mathrm{G}+\mathrm{C} \%)$. Among the 47 Isodon spp. (including I. rugosus), the spanning length of ITS-2 regions was partial in 15 species (193 nt in 3 sp., 194 nt in 7 sp., $195 \mathrm{nt}$ in 1 sp., $197 \mathrm{nt}$ in 2 sp., $199 \mathrm{nt}$ in $1 \mathrm{sp} ., 205 \mathrm{nt}$ in $1 \mathrm{sp}$.) and complete in 32, excluding I. rugosus, species (207 nt in 4 spp., $208 \mathrm{nt}$ in 20 spp., $209 \mathrm{nt}$ in 4 spp., $210 \mathrm{nt}$ in $1 \mathrm{sp} ., 211 \mathrm{nt}$ in $2 \mathrm{spp}$. and $212 \mathrm{nt}$ in $1 \mathrm{sp}$.) (Table 2).

It was found that only nine species of Isodon: I. amethystoides (I-4, KF032259.1), I. coetsa (I-8, KM877351.1), I. enanderianus (I-11, JQ389514.1), I. inflexus (I-20, KF032266.1), I. japonicas (I-22, KF032254.1), I. megathyrsus (I-27, MH117581.1), I. nervosus (I-29, KF032257.1), I. rubescens (I-36, KF032249.1) and I. serra (I-40, KF032262.1) were defined previously as complete for all the three regions of $n r D N A-I T S$ (i.e. ITS-1 + 5.8S + ITS-2) by their respective authors in NCBI database (Table 1). By taking reference to the similar definition or defined regions of these nine taxa, only 32 species (I-01, I-03, I-04, I-07, I-08, I-11, I-12, I-13, 
Table 2. Defined length (L), spanning region $(>)$ and nucleotide composition $(\mathrm{G}+\mathrm{C} \%$ ) of the ITS-1, 5.8S, ITS-2 regions of Isodon rugosus and related species

\begin{tabular}{|c|c|c|c|c|c|c|}
\hline \multirow{2}{*}{ Accession No. } & \multicolumn{2}{|l|}{ ITS-1 } & \multicolumn{2}{|l|}{$5.8 S$} & \multicolumn{2}{|l|}{ ITS-2 } \\
\hline & $\mathrm{L}>$ region & $\mathrm{G}+\mathrm{C} \%$ & $\mathrm{~L}>$ region & $\mathrm{G}+\mathrm{C} \%$ & $\mathrm{~L}>$ region & $\mathrm{G}+\mathrm{C} \%$ \\
\hline MH931390 (I-1) & $198>39 \ldots .236$ & 66.16 & $164>237 \ldots . .400$ & 54.88 & $207>401 \ldots .607$ & 68.12 \\
\hline FJ593355.1 (I-2) & $196>01 \ldots . .196$ & 63.78 & $164>197 \ldots . .360$ & 54.88 & $205>361 \ldots . .565$ & 65.85 \\
\hline FJ593356.1 (I-3) & $196>01 \ldots \ldots 196$ & 65.82 & $164>197 \ldots . .360$ & 54.88 & $208>361 \ldots . .568$ & 67.79 \\
\hline KF032259.1 (I-4) & $196>15 \ldots . .210$ & 65.82 & $164>211 \ldots . .374$ & 54.88 & $207 *>375 \ldots . .567$ & 67.15 \\
\hline KF855420.1 (I-5) & $196>01 \ldots \ldots 196$ & 64.80 & $164>197 \ldots . .360$ & 54.88 & $197>361 \ldots . .557$ & 69.04 \\
\hline KF855427.1 (I-6) & $194>01 \ldots \ldots 194$ & 65.46 & $164>195 \ldots \ldots 358$ & 54.88 & $194 * * *>359 \ldots . .552$ & 68.04 \\
\hline FJ593358.1 (I-7) & $196>01 \ldots . .196$ & 64.29 & $164>197 \ldots . .360$ & 54.88 & $211>361 \ldots . .571$ & 68.72 \\
\hline KM877351.1 (I-8) & $195>33 \ldots .227$ & 64.62 & $164>228 \ldots . .391$ & 55.49 & $208>392 \ldots . .599$ & 66.35 \\
\hline KF855429.1 (I-9) & $196>01 \ldots . .196$ & 65.31 & $164>197 \ldots . .360$ & 54.88 & $194>361 \ldots . .554$ & 68.56 \\
\hline KF855444.1 (I-10) & $196 *>01 \ldots \ldots 196$ & 64.79 & $164>197 \ldots \ldots 360$ & 54.88 & $194 *>361 \ldots . .554$ & 67.53 \\
\hline JQ389514.1 (I-11) & $196>06 \ldots . .201$ & 65.82 & $164>202 \ldots \ldots 365$ & 54.88 & $208>366 \ldots . .573$ & 66.35 \\
\hline FJ593364.1 (I-12) & $196 * *>01 \ldots \ldots 196$ & 64.29 & $164>197 \ldots . .360$ & 54.88 & $208 *>361 \ldots . .568$ & 67.31 \\
\hline FJ593365.1 (I-13) & $196>01 \ldots \ldots 196$ & 64.80 & $164>197 \ldots \ldots 360$ & 54.88 & $212>361 \ldots \ldots 572$ & 66.03 \\
\hline FJ593366.1 (I-14) & $196>01 \ldots \ldots 196$ & 65.82 & $164>197 \ldots \ldots 360$ & 54.88 & $208>361 \ldots . .568$ & 66.83 \\
\hline FJ593367.1 (I-15) & $196>01 \ldots .196$ & 65.82 & $164>197 \ldots . .360$ & 54.88 & $208>361 \ldots . .568$ & 67.79 \\
\hline FJ593368.1 (I-16) & $196^{*}>01 \ldots \ldots 196$ & 65.82 & $164>197 \ldots . .360$ & 54.88 & $208>361 \ldots . .568$ & 68.27 \\
\hline FJ593369.1 (I-17) & $196>01 \ldots \ldots 196$ & 65.31 & $164>197 \ldots \ldots 360$ & 54.88 & $209>361 \ldots . .569$ & 67.94 \\
\hline FJ593370.1 (I-18) & $196 *>01 \ldots \ldots 196$ & 65.31 & $164>197 \ldots \ldots 360$ & 54.88 & $209>361 \ldots . .569$ & 67.94 \\
\hline FJ593371.1 (I-19) & $196>01 \ldots \ldots 196$ & 64.80 & $164>197 \ldots \ldots 360$ & 54.88 & $208>361 \ldots . .568$ & 67.79 \\
\hline KF032266.1 (I-20) & $196>15 \ldots \ldots 210$ & 64.80 & $164>211 \ldots . .374$ & 54.27 & $211>375 \ldots . .585$ & 67.77 \\
\hline KF855439.1 (I-21) & $196^{* * *}>01 \ldots \ldots 196$ & 63.78 & $164>197 \ldots . .360$ & 54.88 & $194 *>361 \ldots . .554$ & 68.56 \\
\hline KF032254.1 (I-22) & $196>15 \ldots \ldots 210$ & 65.31 & $164>211 \ldots . .374$ & 54.88 & $208>375 \ldots . .582$ & 67.31 \\
\hline KF855421.1 (I-23) & $196>01 \ldots \ldots 196$ & 66.33 & $164>197 \ldots . .360$ & 54.88 & $195>361 \ldots . .555$ & 69.74 \\
\hline KF855445.1 (I-24) & $196>01 \ldots \ldots 196$ & 65.31 & $164>197 \ldots \ldots 360$ & 54.88 & $193>361 \ldots . .553$ & 67.36 \\
\hline FJ593381.1 (I-25) & $196 *>01 \ldots \ldots 196$ & 65.82 & $164>197 \ldots \ldots 360$ & 54.88 & $208>361 \ldots . .568$ & 67.79 \\
\hline FJ593382.1 (I-26) & $196>01 \ldots \ldots 196$ & 65.82 & $164>197 \ldots . .360$ & 55.49 & $208>361 \ldots . .568$ & 67.79 \\
\hline MH117581.1 (I-27) & $196>33 \ldots \ldots 228$ & 64.80 & $164>229 \ldots \ldots 392$ & 54.88 & $208>393 \ldots \ldots 600$ & 67.79 \\
\hline FJ593385.1 (I-28) & $196 *>01 \ldots \ldots 196$ & 65.31 & $164>197 \ldots . .360$ & 54.88 & $208>361 \ldots . .568$ & 67.79 \\
\hline KF032257.1 (I-29) & $196>17 \ldots \ldots 212$ & .33 & $164>213 \ldots . .376$ & 54.88 & $209>377 \ldots . .585$ & 67.94 \\
\hline KF855434.1 (I-30) & $196>01 \ldots \ldots 196$ & .82 & $164>197 \ldots \ldots 360$ & 54.88 & $199 * * * *>361 \ldots \ldots 559$ & 67.34 \\
\hline KF855425.1 (I-31) & $196>01 \ldots \ldots 196$ & & $164>197 \ldots \ldots 360$ & 27 & $197 *>361 \ldots . .557$ & 69.04 \\
\hline KF855430.1 (I-32) & $196^{* * *}>01 \ldots \ldots 196$ & & $164>197 \ldots . .360$ & & $194 *>361 \ldots . .554$ & 68.56 \\
\hline FJ593388.1 (I-33) & $196>01 \ldots \ldots 196$ & 64.29 & $163^{\mathrm{d}}>197 \ldots . .359$ & 55.21 & $208>360 \ldots . .567$ & 67.79 \\
\hline FJ593389.1 (I-34) & $196 * * *>01 \ldots \ldots 196$ & 62.76 & $164>197 \ldots . .360$ & 54.88 & $208 * * *>361 \ldots . .568$ & 66.35 \\
\hline FJ593390.1 (I-35) & $196>01 \ldots \ldots 196$ & 65.31 & $164>197 \ldots \ldots 360$ & 54.88 & $208^{*}>361 \ldots .568$ & 67.31 \\
\hline KF032249.1 (I-36) & $196>17 \ldots \ldots .212$ & 64.80 & $164>213 \ldots . .376$ & 54.88 & $208>377 \ldots . .584$ & 67.79 \\
\hline FJ593391.1 (I-37) & $196>01 \ldots \ldots 196$ & 65.82 & $164>197 \ldots \ldots 360$ & 54.88 & $208>361 \ldots . .568$ & 66.83 \\
\hline FJ593392.1 (I-38) & $196>01 \ldots . .196$ & 65.31 & $164>197 \ldots . .360$ & 54.88 & $208>361 \ldots . .568$ & 67.79 \\
\hline FJ593393.1 (I-39) & $196>01 \ldots \ldots 196$ & 64.80 & $164>197 \ldots \ldots .360$ & 54.88 & $207>361 \ldots . .567$ & 66.67 \\
\hline KF032262.1 (I-40) & $196>17 \ldots \ldots 212$ & 65.81 & $164>213 \ldots . .376$ & 54.88 & $210>377 \ldots . .586$ & 68.10 \\
\hline KF855412.1 (I-41) & $196 *>01 \ldots . .196$ & 65.31 & $164>197 \ldots \ldots 360$ & 54.88 & $194>361 \ldots . .554$ & 69.07 \\
\hline KF855449.1 (I-42) & $196>01 \ldots . .196$ & 64.80 & $164>197 \ldots . .360$ & 54.88 & $193>361 \ldots . .553$ & 68.39 \\
\hline KF855450.1 (I-43) & $196>01 \ldots \ldots 196$ & 64.80 & $164>197 \ldots . .360$ & 55.49 & $193 *>361 \ldots . .553$ & 66.84 \\
\hline FJ593394.1 (I-44) & $196>01 \ldots . .196$ & 65.31 & $164>197 \ldots . .360$ & 54.88 & $209 *>361 \ldots . .569$ & 66.99 \\
\hline FJ593396.1 (I-45) & $196>01 \ldots \ldots 196$ & 64.80 & $164>197 \ldots \ldots 360$ & 54.88 & $208>361 \ldots . .568$ & 67.79 \\
\hline FJ593397.1 (I-46) & $196>01 \ldots \ldots 196$ & 66.33 & $164>197 \ldots \ldots 360$ & 54.88 & $207>361 \ldots . .567$ & 67.15 \\
\hline KF855436.1 (I-47) & $196>01 \ldots \ldots 196$ & 65.82 & $164>197 \ldots . .360$ & 54.88 & $194 * *>361 \ldots . .554$ & 68.56 \\
\hline
\end{tabular}

$\mathrm{L}$ - length in nucleotide bases, $\mathrm{N}$-any nucleotide base, ${ }^{*}-\mathrm{N}(1),{ }^{* *}-\mathrm{N}(2) ; * * *-\mathrm{N}(3), ; * * * \mathrm{~N}(4), \mathrm{d}-\operatorname{deletion} 1$. 
Table 3. Distribution of informative (PI) sites and nucleotide base positions in the aligned ITS-1 region of Isodon rugosus

\begin{tabular}{|c|c|c|c|c|c|c|c|c|c|c|c|c|c|c|c|c|c|c|c|}
\hline \multirow{2}{*}{ Species* } & \multicolumn{19}{|c|}{ ITS-1 region $(>39 \ldots . .236)$} \\
\hline & 60 & 67 & 77 & 82 & 84 & 94 & 100 & 106 & 114 & 116 & 147 & 166 & 167 & 180 & 183 & 205 & 213 & 221 & 229 \\
\hline $\mathrm{I}-01$ & $\mathrm{C}$ & A & $\mathrm{C}$ & $\mathrm{T}$ & $\mathrm{C}$ & A & $\mathrm{C}$ & $\mathrm{C}$ & G & $\mathrm{G}$ & $\mathrm{A}$ & A & $\mathrm{T}$ & $\mathrm{C}$ & $\mathrm{C}$ & $\mathrm{G}$ & $\mathrm{C}$ & $\mathrm{G}$ & $\mathrm{C}$ \\
\hline $\mathrm{I}-02$ & - & - & - & - & - & - & - & - & A & $\mathrm{T}$ & $\mathrm{C}$ & - & - & - & - & - & - & A & - \\
\hline $\mathrm{I}-03$ & - & - & - & - & - & - & - & - & - & $\mathrm{T}$ & $\mathrm{C}$ & - & - & - & - & - & - & - & - \\
\hline $\mathrm{I}-04$ & - & - & - & - & - & - & - & - & - & - & $\mathrm{C}$ & - & - & - & - & - & - & - & A \\
\hline $\mathrm{I}-05$ & - & - & - & - & - & - & $\mathrm{T}$ & - & - & - & $\mathrm{C}$ & - & - & - & - & $\mathrm{T}$ & - & - & - \\
\hline $\mathrm{I}-06$ & - & - & - & - & - & - & $* *$ & - & - & $\mathrm{T}$ & $\mathrm{C}$ & - & - & - & - & - & - & - & - \\
\hline $\mathrm{I}-07$ & $\mathrm{~T}$ & - & - & - & - & - & - & - & - & - & $\mathrm{C}$ & - & - & - & - & $\mathrm{T}$ & - & - & $\mathrm{T}$ \\
\hline $\mathrm{I}-08$ & $\mathrm{~T}$ & - & - & - & - & - & - & - & - & - & $\mathrm{C}$ & - & - & - & - & - & - & - & - \\
\hline I-09 & - & - & - & - & - & - & - & - & - & $\mathrm{T}$ & $\mathrm{T}$ & - & - & - & - & - & - & - & - \\
\hline $\mathrm{I}-10$ & - & - & - & A & - & - & - & - & - & - & $\mathrm{C}$ & - & - & - & A & - & - & - & A \\
\hline $\mathrm{I}-11$ & - & - & - & - & - & $\mathrm{G}$ & - & - & - & A & $\mathrm{C}$ & - & - & - & - & - & - & A & - \\
\hline $\mathrm{I}-12$ & - & - & - & - & - & $\mathrm{G}$ & - & - & - & $\mathrm{T}$ & - & - & - & $* *$ & - & - & - & - & - \\
\hline $\mathrm{I}-13$ & - & - & - & - & - & - & - & - & - & $\mathrm{T}$ & $\mathrm{C}$ & - & - & - & - & - & - & - & - \\
\hline I-14 & - & - & - & - & - & - & - & - & - & $\mathrm{T}$ & $\mathrm{C}$ & - & - & - & - & - & - & - & - \\
\hline $\mathrm{I}-15$ & - & - & - & - & - & - & - & - & - & - & - & - & - & - & - & - & - & - & - \\
\hline I-16 & - & - & - & - & - & - & - & - & - & $* *$ & $\mathrm{C}$ & - & - & - & - & - & - & - & - \\
\hline I-17 & - & - & - & - & - & - & - & - & - & - & $\mathrm{C}$ & - & - & $\mathrm{T}$ & - & - & - & - & A \\
\hline $\mathrm{I}-18$ & - & - & - & - & - & - & - & - & - & $* *$ & $\mathrm{C}$ & - & - & - & - & - & - & - & - \\
\hline I-19 & - & - & - & - & - & - & - & - & $\mathrm{T}$ & $\mathrm{T}$ & - & - & - & - & - & - & - & - & - \\
\hline $\mathrm{I}-20$ & - & - & - & - & $\mathrm{T}$ & - & - & - & - & - & $\mathrm{C}$ & $\mathrm{T}$ & - & - & A & - & - & - & A \\
\hline I-21 & - & - & A & - & - & - & - & - & - & $\mathrm{T}$ & $\mathrm{C}$ & - & - & - & - & - & - & - & $* *$ \\
\hline I-22 & - & - & - & - & - & - & - & - & - & - & $\mathrm{C}$ & - & - & - & - & - & - & - & A \\
\hline I -23 & - & - & - & - & - & - & - & - & - & - & $\mathrm{C}$ & - & - & - & - & - & - & - & - \\
\hline I-24 & - & - & - & - & $\mathrm{T}$ & - & - & - & - & - & $\mathrm{C}$ & $\mathrm{T}$ & - & - & - & A & - & - & - \\
\hline $\mathrm{I}-25$ & - & $\mathrm{T}$ & - & - & - & - & - & - & - & - & $\mathrm{C}$ & - & - & - & - & - & - & $* *$ & - \\
\hline I-26 & - & - & - & - & - & - & - & - & - & $\mathrm{T}$ & $\mathrm{C}$ & - & - & - & - & - & - & - & - \\
\hline $\mathrm{I}-27$ & - & - & - & - & - & - & - & A & - & - & $\mathrm{C}$ & - & - & - & - & - & - & - & - \\
\hline I-28 & - & $\mathrm{T}$ & - & A & - & - & - & - & - & $* *$ & $\mathrm{C}$ & - & - & - & - & - & - & - & - \\
\hline I-29 & - & - & - & - & - & - & - & - & - & - & $\mathrm{C}$ & - & - & - & - & - & - & - & - \\
\hline I-30 & - & - & - & - & - & - & - & - & - & $\mathrm{T}$ & $\mathrm{C}$ & - & - & - & - & - & - & - & - \\
\hline I-31 & - & - & - & - & - & - & - & - & - & - & $\mathrm{C}$ & - & - & - & - & - & - & - & - \\
\hline I-32 & - & - & $\mathrm{T}$ & - & - & - & - & - & $\mathrm{T}$ & $\mathrm{T}$ & $* *$ & - & - & - & - & - & - & - & - \\
\hline I-33 & - & - & - & - & - & - & - & - & - & - & $\mathrm{T}$ & - & - & - & - & - & - & - & - \\
\hline I-34 & - & - & - & - & - & - & $\mathrm{Y}$ & - & - & - & $\mathrm{C}$ & - & - & - & - & - & $\mathrm{T}$ & - & - \\
\hline $\mathrm{I}-35$ & - & - & - & - & - & - & - & - & - & - & $\mathrm{C}$ & - & - & - & - & - & $\mathrm{T}$ & - & A \\
\hline I-36 & - & - & - & - & - & - & - & - & - & A & $\mathrm{C}$ & - & - & - & - & - & $\mathrm{T}$ & - & A \\
\hline I-37 & - & $\mathrm{T}$ & - & - & - & - & - & - & - & - & $\mathrm{C}$ & - & - & - & - & - & - & - & - \\
\hline I-38 & - & - & - & - & - & - & - & A & - & - & $\mathrm{C}$ & - & - & $\mathrm{T}$ & - & - & - & - & - \\
\hline I-39 & - & - & - & - & - & - & - & - & - & $\mathrm{T}$ & $\mathrm{C}$ & - & - & - & - & - & - & - & - \\
\hline $\mathrm{I}-40$ & - & - & - & - & - & - & - & - & - & - & $\mathrm{C}$ & - & - & - & - & - & - & - & - \\
\hline I -41 & - & - & A & - & - & - & - & - & - & - & $\mathrm{C}$ & - & - & - & - & - & - & - & - \\
\hline I -42 & - & - & - & - & - & - & - & - & - & - & - & $\mathrm{T}$ & A & $\mathrm{T}$ & - & - & - & - & - \\
\hline I -43 & - & - & - & - & $\mathrm{T}$ & - & $\mathrm{T}$ & - & - & - & $\mathrm{C}$ & $\mathrm{T}$ & A & - & - & A & - & - & - \\
\hline $\mathrm{I}-44$ & - & - & - & - & - & - & $\mathrm{T}$ & - & - & - & $\mathrm{C}$ & - & - & - & - & - & - & A & - \\
\hline $\mathrm{I}-45$ & - & - & - & - & - & - & - & - & $\mathrm{T}$ & $\mathrm{T}$ & $\mathrm{C}$ & - & - & - & - & - & - & - & - \\
\hline $\mathrm{I}-46$ & - & - & - & - & - & - & - & - & - & - & $\mathrm{C}$ & - & - & - & - & - & - & - & - \\
\hline $\mathrm{I}-47$ & - & - & - & - & - & - & - & - & - & $\mathrm{T}$ & $\mathrm{C}$ & - & - & - & - & - & - & - & - \\
\hline
\end{tabular}

*Abbreviation of species see in Table 1, '-'- bars represents the identical nucleotides as that of I. rugosus in their respectively aligned sequence sites, $* * \mathrm{~N}-$ any nucleotide base. 
Table 4. Distribution of informative (PI) sites and nucleotide base positions in the aligned 5.8S $n r D N A$ and ITS-2 region of Isodon rugosus

\begin{tabular}{|c|c|c|c|c|c|c|c|c|c|c|c|c|c|}
\hline \multirow{2}{*}{ Species* } & \multirow{2}{*}{\begin{tabular}{|c|}
$5.8 S$ region $(>237 \ldots . .400)$ \\
237
\end{tabular}} & \multicolumn{12}{|c|}{ ITS-2 region $(>401 \ldots \ldots 607)$} \\
\hline & & 418 & 419 & 424 & 425 & 429 & 433 & 450 & 502 & 551 & 558 & 565 & 568 \\
\hline $\mathrm{I}-01$ & $\mathrm{~A}$ & A & $\mathrm{C}$ & $\mathrm{G}$ & $\mathrm{C}$ & $\mathrm{C}$ & $\mathrm{G}$ & $\mathrm{T}$ & $\mathrm{C}$ & $\mathrm{C}$ & $\mathrm{T}$ & $\mathrm{T}$ & $\mathrm{C}$ \\
\hline $\mathrm{I}-02$ & - & - & - & - & $\mathrm{T}$ & - & - & - & - & gp & $\mathrm{C}$ & - & $\mathrm{T}$ \\
\hline $\mathrm{I}-03$ & - & $\mathrm{C}$ & A & $\mathrm{T}$ & - & - & - & - & - & - & $\mathrm{C}$ & - & $\mathrm{T}$ \\
\hline $\mathrm{I}-04$ & - & $\mathrm{C}$ & A & $\mathrm{T}$ & - & - & - & - & - & - & $\mathrm{C}$ & - & $\mathrm{T}$ \\
\hline $\mathrm{I}-05$ & - & $\mathrm{C}$ & A & $\mathrm{C}$ & - & - & - & - & - & - & $\mathrm{C}$ & - & - \\
\hline I-06 & - & $\mathrm{C}$ & A & $* *$ & - & - & - & - & - & - & C & - & - \\
\hline $\mathrm{I}-07$ & - & $\mathrm{C}$ & A & $\mathrm{C}$ & - & - & - & - & - & - & C & - & - \\
\hline $\mathrm{I}-08$ & $\mathrm{G}$ & - & - & - & - & - & - & - & - & - & - & - & $\mathrm{T}$ \\
\hline $\mathrm{I}-09$ & - & $\mathrm{C}$ & A & $\mathrm{T}$ & - & - & - & - & - & - & $\mathrm{C}$ & - & - \\
\hline $\mathrm{I}-10$ & - & C & A & $\mathrm{T}$ & - & $* *$ & - & - & - & - & $\mathrm{C}$ & - & $\mathrm{T}$ \\
\hline $\mathrm{I}-11$ & - & $\mathrm{C}$ & A & $\mathrm{T}$ & - & - & - & - & $\mathrm{T}$ & $\mathrm{T}$ & $\mathrm{C}$ & - & $\mathrm{T}$ \\
\hline $\mathrm{I}-12$ & - & $\mathrm{C}$ & A & $\mathrm{T}$ & - & - & - & - & - & - & $\mathrm{C}$ & - & $\mathrm{T}$ \\
\hline $\mathrm{I}-13$ & - & $\mathrm{C}$ & - & $\mathrm{C}$ & - & - & - & - & - & $\mathrm{T}$ & $\mathrm{C}$ & - & $\mathrm{T}$ \\
\hline I-14 & - & $\mathrm{C}$ & A & $\mathrm{T}$ & $\mathrm{T}$ & - & - & - & - & - & $\mathrm{C}$ & $\mathrm{C}$ & $\mathrm{T}$ \\
\hline $\mathrm{I}-15$ & - & $\mathrm{C}$ & A & $\mathrm{T}$ & - & - & - & - & - & - & $\mathrm{C}$ & - & $\mathrm{T}$ \\
\hline I-16 & - & C & A & $\mathrm{T}$ & - & - & - & - & - & - & $\mathrm{C}$ & $\mathrm{C}$ & $\mathrm{T}$ \\
\hline $\mathrm{I}-17$ & - & $\mathrm{C}$ & - & $\mathrm{T}$ & - & - & - & - & - & - & $\mathrm{C}$ & - & $\mathrm{T}$ \\
\hline I-18 & - & $\mathrm{C}$ & A & $\mathrm{T}$ & - & - & - & $\mathrm{C}$ & - & - & $\mathrm{C}$ & - & $\mathrm{T}$ \\
\hline $\mathrm{I}-19$ & - & $\mathrm{C}$ & A & $\mathrm{T}$ & - & - & - & - & - & - & $\mathrm{C}$ & - & $\mathrm{T}$ \\
\hline $\mathrm{I}-20$ & - & - & - & $\mathrm{T}$ & - & A & - & - & - & - & $\mathrm{C}$ & - & $\mathrm{T}$ \\
\hline $\mathrm{I}-21$ & - & $\mathrm{C}$ & A & $\mathrm{T}$ & - & - & - & - & - & - & $\mathrm{C}$ & - & $\mathrm{T}$ \\
\hline $\mathrm{I}-22$ & - & $\mathrm{C}$ & A & $\mathrm{T}$ & - & - & - & - & - & - & C & - & $\mathrm{T}$ \\
\hline I-23 & - & $\mathrm{C}$ & - & $\mathrm{T}$ & - & - & - & - & - & - & C & C & - \\
\hline I-24 & - & - & A & - & $\mathrm{T}$ & - & - & - & $\mathrm{T}$ & - & $\mathrm{C}$ & - & $\mathrm{T}$ \\
\hline $\mathrm{I}-25$ & - & $\mathrm{C}$ & A & $\mathrm{T}$ & - & - & - & - & - & - & $\mathrm{C}$ & - & $\mathrm{T}$ \\
\hline $\mathrm{I}-26$ & G & C & A & $\mathrm{T}$ & - & - & - & - & - & - & C & C & $\mathrm{T}$ \\
\hline $\mathrm{I}-27$ & - & $\mathrm{C}$ & $\mathrm{A}$ & $\mathrm{T}$ & - & - & A & - & - & - & $\mathrm{C}$ & $\mathrm{C}$ & $\mathrm{T}$ \\
\hline I-28 & - & C & A & $\mathrm{T}$ & - & - & - & - & - & - & $\mathrm{C}$ & - & $\mathrm{T}$ \\
\hline I-29 & - & C & A & $\mathrm{T}$ & - & - & - & - & - & - & C & - & $\mathrm{T}$ \\
\hline $\mathrm{I}-30$ & - & $\mathrm{C}$ & A & $\mathrm{C}$ & - & - & - & - & - & - & $\mathrm{C}$ & - & $\mathrm{T}$ \\
\hline $\mathrm{I}-31$ & - & $\mathrm{C}$ & A & $\mathrm{C}$ & $* *$ & - & - & - & - & - & $\mathrm{C}$ & $\mathrm{C}$ & $\mathrm{T}$ \\
\hline I-32 & - & $\mathrm{C}$ & A & $\mathrm{T}$ & - & - & - & - & - & - & $\mathrm{C}$ & $* *$ & $\mathrm{~T}$ \\
\hline I-33 & - & $\mathrm{C}$ & A & $\mathrm{T}$ & - & - & - & - & - & - & $\mathrm{C}$ & $\mathrm{C}$ & $\mathrm{T}$ \\
\hline I-34 & - & $\mathrm{C}$ & A & - & - & - & - & - & - & $* *$ & $\mathrm{C}$ & - & $\mathrm{T}$ \\
\hline I-35 & - & $\mathrm{C}$ & A & $\mathrm{T}$ & - & - & - & C & - & $\mathrm{T}$ & $\mathrm{C}$ & - & $\mathrm{T}$ \\
\hline I-36 & - & $\mathrm{C}$ & A & $\mathrm{T}$ & - & - & - & - & - & - & C & - & $\mathrm{T}$ \\
\hline I-37 & - & $\mathrm{C}$ & A & $\mathrm{T}$ & - & A & - & - & - & - & $\mathrm{C}$ & - & $\mathrm{T}$ \\
\hline I-38 & - & $\mathrm{C}$ & A & $\mathrm{T}$ & - & - & A & - & - & - & $\mathrm{C}$ & C & $\mathrm{T}$ \\
\hline I-39 & - & C & A & A & - & - & - & - & - & $\mathrm{T}$ & $\mathrm{C}$ & - & $\mathrm{T}$ \\
\hline $\mathrm{I}-40$ & - & - & $\mathrm{A}$ & $\mathrm{T}$ & - & - & - & - & - & - & $\mathrm{C}$ & - & $\mathrm{T}$ \\
\hline $\mathrm{I}-41$ & - & $\mathrm{C}$ & A & $\mathrm{C}$ & - & - & - & - & - & $\mathrm{T}$ & $\mathrm{C}$ & - & - \\
\hline $\mathrm{I}-42$ & - & - & - & - & $\mathrm{T}$ & - & - & - & $\mathrm{T}$ & - & C & - & $\mathrm{T}$ \\
\hline $\mathrm{I}-43$ & - & - & - & - & $\mathrm{T}$ & - & - & - & $\mathrm{T}$ & - & C & - & $\mathrm{T}$ \\
\hline I-44 & - & - & $\mathrm{gp}$ & $\mathrm{T}$ & - & - & - & - & - & $* *$ & $\mathrm{C}$ & - & $\mathrm{T}$ \\
\hline $\mathrm{I}-45$ & - & $\mathrm{C}$ & $\mathrm{A}$ & $\mathrm{T}$ & - & - & - & - & - & - & - & $\mathrm{C}$ & $\mathrm{T}$ \\
\hline I-46 & - & - & - & - & $\mathrm{T}$ & - & - & - & $\mathrm{T}$ & - & C & - & $\mathrm{T}$ \\
\hline I- 47 & - & $\mathrm{C}$ & A & $\mathrm{T}$ & - & - & - & - & - & - & C & $* *$ & $* *$ \\
\hline
\end{tabular}

*Abbreviation of species see in Table 1, '-' - bars represent the identical nucleotides as that of Isodon rugosus in their respectively aligned sequence sites, $* * \mathrm{~N}-$ any nucleotide base, gp - gap. 
I-14, I-15, I-16, I-17, I-18, I-19, I-20, I-22, I-25, I-26, I-27, I-28, I-29, I-33, I-34, I-35, I-36, I-37, $\mathrm{I}-38, \mathrm{I}-39, \mathrm{I}-40, \mathrm{I}-44, \mathrm{I}-45$ and $\mathrm{I}-46$ ) were found complete in all the three regions of $n r D N A-I T S$ (ITS-1, 5.8S and ITS-2). From the above 32 species, for ITS-2 region, 19 species: I. adenolomus (I-3), I. coetsa (I-8), I. enanderianus (I-11), I. eriocalyx (I-12), I. flavidus (I-14), I. flexicaulis (I-15), I. for- restii (I-16), I. grandifolius var. atuntzeensis (I-19), I. japonicas (I-22), I. loxothyrsus (I-25), I. lungshengensis (I-26), I. megathyrsus (I-27), I. melissoides (I-28), I. phyllostachys (I-33), I. pleiophyllus (I-34), I. rosthornii (I-35), I. rubescens (I-36), I. rugosiformis (I-37), I. taliensis (I-44) possessed similar spanning length of $208 \mathrm{nt}$ (Table 2). Alignment in these taxa reflected a maximum length span of $212 \mathrm{nt}$

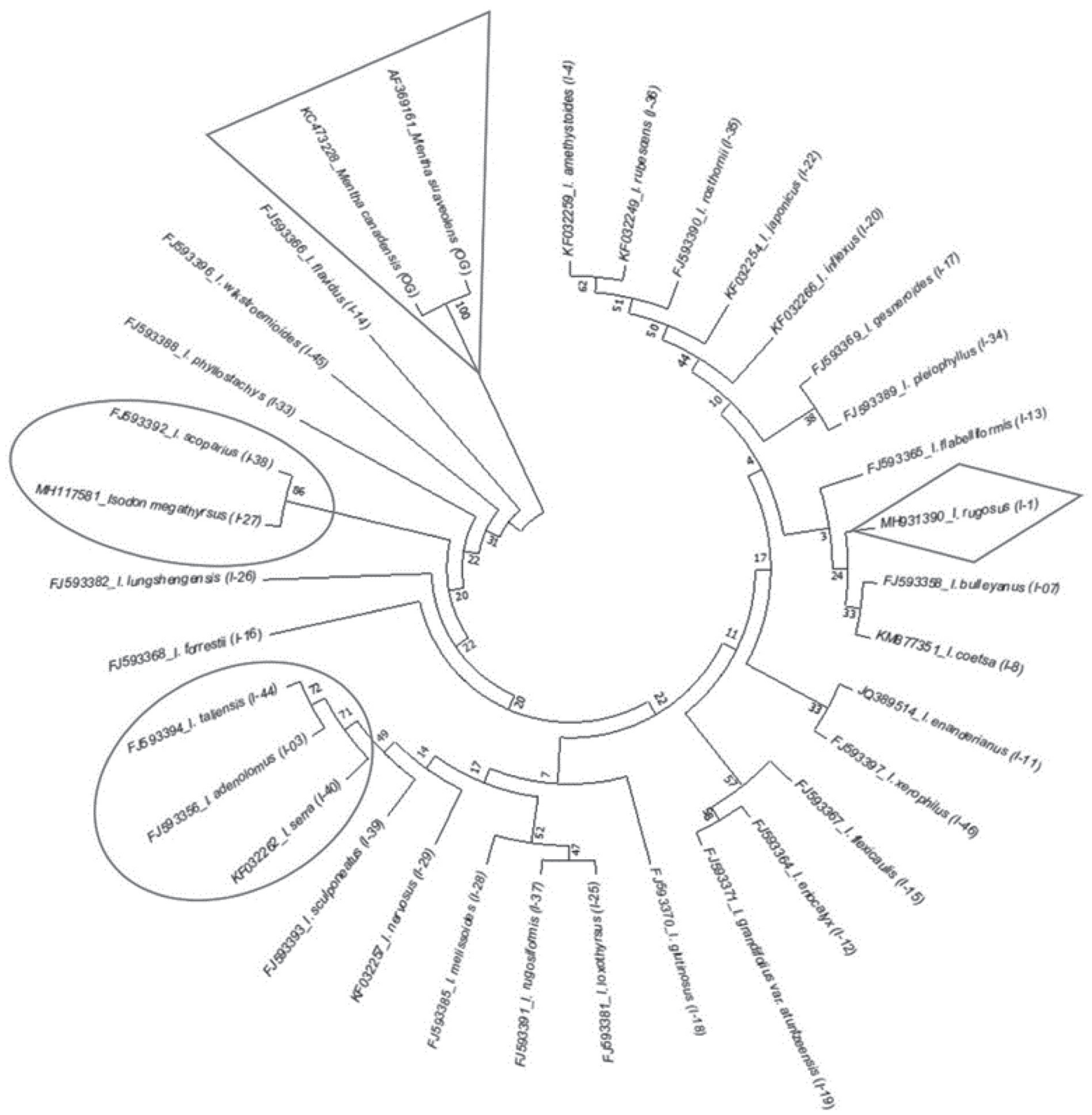

Fig. 1. Phylogenetic tree of Isodon species with two different out-group members (within a triangle), Mentha suaveolensAF369161 and M. canadensis-KC473228, generated using ITS sequence according to the Maximum likelihood (ML) method. Numbers above lines are the bootstrap values in 1000 replicates, species within circles have bootstrap closeness above 70 , query taxon Isodon rugosus within rhombus 
in I. flabelliformis (I-13) and a minimum of $207 \mathrm{nt}$ in four species, namely I. rugosus (I-01), I. amethystoides (I-04), I. sculponeatus (I-39) and I. xerophilus (I-46). Species I. flabelliformis (I-13) was reported with the lowest $(66.03 \%) \mathrm{GC}$ content. It was highest $(68.72 \%)$ in I. bulleyanus (I-07), wherein 11 species, e.g. I. adenolomus (I-03), I. flexicaulis (I-15), I. grandifolius var. atuntzeensis (I-19), I. loxothyrsus (I-25), I. lungshengensis (I-26), I. megathyrsus (I-27), I. melissoides (I-28), I. phyllostachys (I-33), I. rubescens (I-36), I. scoparius (I-38) and I. wikstroemioides (I-45), were reported with same $(67.79 \%)$ GC content.

The 32 species referred above with complete $n r D N A$-ITS regions (ITS- $1+5.8 S+I T S-2)$ were used to check the phylogenetic relationship of I. rugosus. Two different species of genus Mentha were used to confirm the out grouping, namely M. suaveolens (AF369161) and M. canadensis (KC473228). Nucleotide bases counts of nrDNA-ITS in Isodon species was observed with similar trends in their respective regions as $\mathrm{C}>\mathrm{G}>\mathrm{A}>\mathrm{T}$ in ITS $-1, \mathrm{G}>\mathrm{C}>\mathrm{A}>\mathrm{T}$ in $5.8 S$ and $\mathrm{C}>\mathrm{G}>\mathrm{T}>\mathrm{A}$ in ITS-2. The estimation of average evolutionary divergence in overall sequence pairs (i.e. overall mean distance in 34 sequences) was $\mathrm{d} \pm$ S.E. $=0.023 \pm 0.002$ by the p-distance method and $\mathrm{d} \pm$ S.E. $=0.024 \pm 0.002$ by both Jukes-Cantor's (JukES \& CANTOR, 1969) and Kimura 2 parameters (Kimura, 1980).

Phylogenetic tree generated by the maximum likelihood (ML) method (Fig. 1) placed the I. rugosus with a low bootstrap value near to the clade having species I. coesta (I-08) and I. bulleyanus (I-7). It indicated its divergence from the probable most recent common ancestor $(M R C A)$ with missing linked members. The clades obtained above the bootstrap value 50 were informative. Two different clades (a clade having I. scoparius - I. megathyrsus and another having $I$. adenolomus $-I$. taliensis) show relatedness reasonably up to a good extend with their respective $M R C A$ as in both clades bootstrap value was above 73 .

The molecular signature of ITS marker in different medicinal plants has been studied by various researchers (Alice \& CAMPBell, 1999; Doh et al., 2016; Liu et al., 2019; Srivastava \& SAGGOo, 2014). In genus Isodon, 46 species were studied with ITS marker, and they are included in the present study
(Table 1). Similar to the above, species I. rugosus has been studied previously for matK marker by SHINWARI et al. (2018) and Srivastava et al. (2020) and psbA-trnH intergenic spacers by PeKHNA (2019).

Presently, taxon I. rugosus studied for its molecular (nrDNA-ITS) signature showed the presence of 32 informative (PI) sites together in the nrDNA-ITS regions (i.e. ITS- $1+5.8 S+$ ITS- $2=19+1+12$ ). This information could be used as additional taxonomic knowledge for the species to confirm its presence in the local flora.

\section{ACKNOWLEDGEMENTS}

The authors are grateful to the Managing Director and his team of Acme ProGen Biotech (India) Pvt. Ltd. for assisting in molecular and sequencing work.

\section{REFERENCES}

Alice L.A., Campbell C.S., 1999: Phylogeny of $R u$ bus (rosaceae) based on nuclear ribosomal DNA internal transcribed spacer region sequences. American Journal of Botany, 86(1): 81-97.

Bentham G., 1832: Labiatarum: genera et species. James Ridgway and Sons. - London.

Chase M.W., Hills H.H., 1991: Silica Gel: an ideal material for field preservation of leaf samples for DNA studies. - Taxon, 40(2): 215-220.

Chen Y.P., Xiang C.L., Hu G.X., Peng H., 2017: Taxonomic notes on Isodon (Nepetoideae, Lamiaceae) in China I: Revision of four species. Nordic Journal of Botany, 35(2): 162-175.

Cheng T., Xu C., Li L., LI C., Zhang Y., Zhou S., 2015: Barcoding the kingdom plantae: new PCR primers for ITS regions of plants with improved universality and specificity. - Molecular Ecology Resources, 16(1): 138-149.

Doh E.J., Paek S.H., Lee G., Lee M.Y., Оh S.E., 2016: Application of partial internal transcribed spacer sequences for the discrimination of Artemisia capillaris from other Artemisia species. - EvidenceBased Complementary and Alternative Medicine, 2016: 1-12. doi: 10.1155/2016/7043436.

Hollingsworth P.M., 2011: Refining the DNA barcode for land plants. - Proceedings of National Academy of Sciences, 108: 19451-19452.

JuKES T.H., CANTOR C.R., 1969: Evolution of Protein 
Molecules: 21-132. - New York.

Kimura M., 1980: A simple method for estimating evolutionary rates of base substitutions through comparative studies of nucleotide sequences. Journal of Molecular Evolution, 16(2): 111-120.

Kumar S., Stecher G., Tamura K., 2016: MEGA7: Molecular evolutionary genetics analysis version 7.0 for bigger datasets. - Molecular Biology and Evolution, 33(7): 1870-1874.

Liu M., Wang W.G., Sun H.D., Pu J.X., 2017: Diterpenoids from Isodon species: An update. - Natural Product Reports, 34(9): 1090-1140.

Liu Z., Gao Y., Zhou J., 2019: Molecular authentication of the medicinal species of Ligusticum (Ligustici Rhizoma et Radix, "Gao-ben") by integrating non-coding internal transcribed spacer 2 (ITS-2) and its secondary structure. - Frontiers in Plant Science, 10(429): 1-8.

MabBerley D.J. (eds), 2008: Mabberley's plantbook. A portable dictionary of the plants, their classification and uses. - Cambridge.

Peknna B., 2019: In-silico datamining and study of molecular (DNA) barcode in medicinal taxon Isodon rugosus (Lamiaceae) from the flora of Baru Sahib, Himachal Pradesh (India). Master's Thesis, Department of Botany, Eternal University. - India.
Sharma A., 2018: Cyto-morphology, anatomy, phytochemical screening and antimicrobial study in wild Isodon rugosus (syn. Plectranthus rugosus) from Baru-Sahib area of Himachal Pradesh. Master's Thesis, Department of Botany, Eternal University. - India.

Shinwari Z.K., Jan S.A., Khalil A.T., Khan A., Ali M., QAISER M., ZAHRA N.B., 2018: Identification and phylogenetic analysis of selected medicinal plant species from Pakistan: DNA barcoding approach. Pakistan Journal of Botany, 50(2): 553-560.

Spach E., 1840: Histoire naturelle des végétaux IX. Librarie Encyclopédique de Roret. - Paris.

Srivastava D.K., Saggoo M.I.S., 2014: Molecular identification of Verbascum thapsus L. (Ban Tambaaku) and its ITS sequence comparison with other Verbascum L. species. -Medicinal Plants International Journal of Phytomedicines and Related Industries, 6(1): 1-7.

Srivastava D.K., Bansal P., Singh P.K., SaGGOo M.I.S., 2020: Molecular autograph of maturase-k gene in Isodon rugosus (Lamiaceae). - Botanica, 26(1): 95-100.

Zhou J., Wang W., Liu M., Liu Z., 2014: Molecular authentication of the traditional medicinal plant Peucedanum praeruptorum and its substitutes and adulterants by DNA barcoding technique. Pharmacognosy Magazine, 10(40): 385-390.

\section{MOLEKULINIS ISODON RUGOSUS (LAMIACEAE) nrDNA-ITS ŽYMENS PARAŠAS}

\section{Devendra Kumar SRIVASTAVA, Pekhna BANSAL, Pradeep Kumar SINGH, Manjit Inder Singh SAGGOO}

\section{Santrauka}

Vaistinio augalo Isodon rugosus molekulinès nrDNA-ITS sekos buvo ịvertintos, naudojant universaliuosius ITS-1 ir ITS-2 pradmenis. Atlikus in-silico duomenų analizę buvo nustatyta, kad Isodon spp. 46 rūšių ITS sekos atitiko ITS-1, 5.8S ir ITS-2 regionus. Tačiau tik 32 rūšių sekos atitiko visus tris regionus, kitų rūšiu sekos buvo dalinès. Isodon rugosus tyrimas atskleidè penkių genų regionus: $18 \mathrm{~S}$ rRNR (da- linè seka, $>1 \ldots .38$ ), ITS-1 (visa seka, $>39 \ldots .236$ ), 5.8S rRNR (visa seka, $>237 \ldots . .400$ ), ITS-2 (visa seka, $>401 \ldots . .607$ ) ir subgeno 26S rRNR (dalinè seka, $>608 \ldots$.. 672). Sekos regionai buvo nustatyti kintamose, singletoninèse ir rūšims būdingose informatyviose srityse. Informacija buvo pateikta filogenetiniame medyje, sudarytame naudojant maksimalios tikimybès metodą. 